\title{
ZERO SETS FOR CONTINUOUS FUNCTIONS ${ }^{1}$
}

\author{
R. C. BUCK
}

1. Introduction. Let $D$ be a closed set in $m$-space, and let $V$ be a linear space of real valued continuous functions defined on $D$. For any $f \in V$, let $Z(f)$ be its zero-set, the set of $p \in D$ with $f(p)=0$. If $m=1$, and $V$ is a class of well behaved functions, then $Z(f)$ will be discrete except for $f$ identically zero. This behavior depends strongly upon $m$. When $m \geqq 2$, then-except in special cases-one expects $Z(f)$ to be a nowhere dense set which is locally of dimension $m-1$.

In this note, we shall be concerned with certain distribution-properties of the zero-sets for functions of $m$ variables which do not seem to have been explicitly stated before; as will be seen, they do not depend upon the nature of the functions in $V$ but only upon the dimension of $V$. If $\operatorname{dim}(V)=N$, then there exists a system of $N$ nonempty disjoint relatively open sets $D_{1}, D_{2}, \cdots, D_{N}$ in $D$ such that if $f$ and $g$ are in $V$, and if they agree at some point in each of the sets $D_{j}$, then $f=g$. Otherwise stated, if $f \in V$ and $Z(f)$ intersects each of the sets $D_{j}$, then $f \equiv 0$.

If $D$ is the plane, and $V$ the class of functions $f(x, y)=a x+b y+c$, then the assertion is merely that there are three open sets $D_{j}$ which cannot all be cut by a single straight line. Again, choose $V$ as the space of polynomials in $x, y, z$ of degree at most 2 . If $f \in V$ but $f \not \equiv 0$ then $Z(f)$ is a quadric surface in 3-space. Any set of nine points in 3 -space lies in some set $Z(f)$; most sets of ten points do not. If we choose a set of points $p_{1}, \cdots, p_{10}$ through which no quadric passes, and then surround each by a sufficiently small open sphere, we obtain ten open sets $D_{1}, \cdots, D_{10}$ which form a system of uniqueness domains for the space $V$.

2. Uniqueness domains. Let $V$ be any subspace of $C[D]$ of dimension $N$, and let $\phi_{1}, \phi_{2}, \cdots, \phi_{N}$ be a basis for $V$.

TheOREM 1. There exist disjoint nonempty sets $D_{1}, D_{2}, \cdots, D_{N}$, relatively open in $D$, and a constant $B$ such that for any $f \in V$, and $p \in D$, and any choice of $p_{i} \in \bar{D}_{i}$

$$
|f(p)| \leqq B\left\{\left|f\left(p_{1}\right)\right|+\left|f\left(p_{2}\right)\right|+\cdots+\left|f\left(p_{N}\right)\right|\right\} .
$$

Proof. Let $S$ be the set of all points in $N$-space of the form $x=\left\langle\phi_{1}(p), \phi_{2}(p), \cdots, \phi_{N}(p)\right\rangle$ for $p \in D$. If $S$ were to lie in a proper

Received by the editors August 10, 1959 and, in revised form, September 23, 1959.

1 This work was carried out under OOR Contract DA-11-022-ORD-2059. 
subspace of $N$-space, there would exist constants $b_{1}, b_{2}, \cdots, b_{N}$ such that $\sum b_{j} x_{j}=0$ for all $x \in S$. This would imply that $\sum b_{j} \phi_{j}=0$ so that the functions $\phi_{j}$ would not have been independent. We can therefore assume that $S$ generates all of $N$-space. There then exist points $p_{1}, p_{2}, \cdots, p_{N}$ in $D$ such that the $N$ points $\left\langle\phi_{1}\left(p_{i}\right), \phi_{2}\left(p_{i}\right), \cdots, \phi_{N}\left(p_{i}\right)\right\rangle$, for $i=1,2, \cdots, N$, are linearly independent. Accordingly, the $N$ by $N$ matrix

$$
\Delta=\left(\begin{array}{cccc}
\phi_{1}\left(p_{1}\right) & \phi_{2}\left(p_{1}\right) & \cdots & \phi_{N}\left(p_{1}\right) \\
\phi_{1}\left(p_{2}\right) & \phi_{2}\left(p_{2}\right) & \cdots & \phi_{N}\left(p_{2}\right) \\
\vdots & \vdots & & \vdots \\
\phi_{1}\left(p_{N}\right) & \phi_{2}\left(p_{N}\right) & \cdots & \phi_{N}\left(p_{N}\right)
\end{array}\right)
$$

is nonsingular.

For any point $p \in D$, let $L_{p}$ be the functional defined on $C[D]$ by $L_{p}(f)=f(p)$. The functionals $L_{p_{i}}$, for $i=1,2, \cdots, N$, are linearly independent; for, if $\sum c_{i} L_{p_{i}}=0$, then for each $j=1,2, \cdots, N$, we would have

$$
0=\sum c_{i} L_{p_{i}}\left(\phi_{j}\right)=\sum c_{i} \phi_{j}\left(p_{i}\right)
$$

and by the nonsingularity of $\Delta, c_{1}=c_{2}=\cdots=0$. Since $\operatorname{dim}(V)=N$, its dual space $V^{\prime}$ is also of dimension $N$. Accordingly, $L_{p_{1}}, L_{p_{2}}, \cdots$, $L_{p_{N}}$ constitutes a basis for $V^{\prime}$. In particular then, if $f \in V$ and $L_{p_{i}}(f)$ $=f\left(p_{i}\right)=0$ for $i=1,2, \cdots, N$, then $L(f)=0$ for every $L \in V^{\prime}$, and $f \equiv 0$. This argument rests upon the nonsingularity of $\Delta$; since the functions $\phi_{1}, \phi_{2}, \cdots, \phi_{N}$ are continuous on $D, d\left(p_{1}, p_{2}, \cdots, p_{N}\right)$ $=\operatorname{det}(\Delta)$ is a continuous function on $D^{N}=D \times D \times \cdots \times D$. In particular, we can choose nonempty open sets $D_{1}, D_{2}, \cdots, D_{N}$ in $D$ so that $d\left(p_{1}, p_{2}, \cdots, p_{N}\right) \neq 0$ for $p_{i} \in D_{i}$. As before, the functionals $L_{p_{1}}, L_{p_{2}}, \cdots, L_{p_{N}}$ will form a basis for $V^{\prime}$ for any choices of the points $p_{i}$, with $p_{1} \in D_{1}, p_{2} \in D_{2}, \cdots, p_{N} \in D_{N}$. Hence, if $f \in V$, and $Z(f)$ touches each of the sets $D_{i}, i=1,2, \cdots, N$, then $f \equiv 0$. This is part of the statement (1). To obtain the more complete formulation, we observe that the sets $D_{i}$ can be taken so that inf $\left|d\left(p_{1}, \cdots, p_{N}\right)\right|$. $=\delta>0$, for all $p_{i} \in \bar{D}_{i}, i=1,2, \cdots, N$. Let $P=\left(p_{1}, p_{2}, \cdots, p_{N}\right)$, and let $C_{j}(r, P)$ be the unique numbers defined by

$$
\sum_{j=1}^{N} C_{j}(r, P) \phi_{j}\left(p_{k}\right)= \begin{cases}1 & \text { if } k=r \\ 0 & \text { if } k \neq r\end{cases}
$$

for $r=1,2, \cdots, N$, and any $P$ in $D_{1} \times D_{2} \times \cdots \times D_{N}$. For each $j$ and $r, C_{j}(r, P)$ is continuous in $P$, and since $|d(P)| \geqq \delta$, there exists a constant $B_{0}$, determined by the functions $\left\{\phi_{j}\right\}$ and the sets $D_{i}$, such that 


$$
\left|C_{j}(r, P)\right| \leqq B_{0}
$$

Setting $\psi_{r}=\sum_{j=1}^{N} C_{j}(r, P) \phi_{j}$, we obtain a new basis for $V$ such that for any $f \in V$,

$$
f=\sum_{r=1}^{N} f\left(p_{r}\right) \psi_{r}
$$

Moreover, for any $r$,

$$
\left\|\psi_{r}\right\| \leqq \sum_{1}^{N}\left|C_{j}(r, P)\right|\left\|\phi_{j}\right\| \leqq B_{0} \sum_{1}^{N}\left\|\phi_{j}\right\| \leqq B
$$

where we have used $\|g\|$ to denote $\sup _{q \in D}|g(q)|$, for any $g \in C[D]$. Note that $B$ again is determined only by the $\left\{\phi_{j}\right\}$ and the $D_{i}$. Returning to (2), we see that

$$
\|f\| \leqq B \sum_{1}^{N}\left|f\left(p_{i}\right)\right|
$$

holding for any choice of $p_{i} \in D_{i}$, for $i=1,2, \cdots, N$.

The conclusion of Theorem 1 can be restated in an interesting way. Introduce a special functional $M$ on $C[D]$ :

$$
M(f)=\sum_{1}^{N} \min _{p_{i} \in \bar{D}_{i}}\left|f\left(p_{i}\right)\right| .
$$

If we were to replace "min" by "max," this would define a seminorm on $C[D]$. On the finite dimensional subspace $V$, it would in fact be a norm, and would therefore have to define there the unique locally convex linear topology. As defined above, however, $M$ is not a seminorm, for it is not true in general that $M(f+g) \leqq M(f)+M(g)$. However, Theorem 1 implies that $M$ still defines a Hausdorff topology on $V$; if $M(f)=0$, then $f$ must take the value zero somewhere in each of the sets $\bar{D}_{i}$, and $f \equiv 0$. The essence of the more general formulation (1) is that this topology again coincides with the unique linear topology in $V$, and there must exist a constant $B$ such that $\|f\|$ $\leqq B M(f)$, for all $f \in V$.

Another familiar instance of this general principle is the fact that in a finite dimensional function space, uniform convergence and $L^{1}$ (or $L^{p}$ ) convergence coincide. This is usually formulated as an inequality: there is a constant $A$ such that

$$
|f(p)| \leqq A \int_{D}|f|
$$


for all $p \in D$, and $f \in V$. This follows at once from (1). Let $\delta$ be the smallest of the measures $\mu\left(D_{i}\right)$. Then,

$$
\int_{D}|f| \geqq \sum_{1}^{N} \int_{D_{i}}|f| \geqq \sum_{1}^{N} \mu\left(D_{i}\right) \min _{p_{i} \in \bar{D}_{i}}\left|f\left(p_{i}\right)\right| \geqq \delta M(f) \geqq \frac{\delta}{B}\|f\| .
$$

3. Generalizations. It is natural to ask if there are any other necessary restrictions upon the sets $\left\{D_{i}\right\}$ if they are to be uniqueness domains. This is answered by the next statement.

Theorem 2. If $D_{1}, D_{2}, \cdots, D_{N}$ is any collection of open disjoint nonempty subsets of $D$, then there is a subspace $V$ of dimension $N$ in $C[D]$ such that if $f \in V$, and if $Z(f)$ touches each of the sets $D_{i}$, then $f \equiv 0$.

Choose functions $\phi_{i}$ so that $\phi_{i}(p)$ is the distance from $p$ to the closed complement of $D_{i}$. Then, $\phi_{i}$ vanishes off $D_{i}$, but is strictly positive on $D_{i}$. Let $V$ be the set of functions $f=\sum c_{i} \phi_{i}$. If $f$ takes the value 0 at some point of $D_{i}$, then it follows that $c_{i}=0$; accordingly, if $Z(f)$ contains at least one point of each $D_{i}$, then $f \equiv 0$.

From this, by a method suggested by the referee, other examples can be constructed. Let $\theta$ be any continuous mapping of $D$ onto another set $D^{\prime}$, and let $V^{\prime}$ be an $N$ dimensional subspace of $C\left[D^{\prime}\right]$. Suppose that $\left\{D_{1}^{\prime}, \cdots, D_{N}^{\prime}\right\}$ is a collection of uniqueness domains in $D^{\prime}$, and let $D_{i}=\theta^{-1}\left(D_{i}^{\prime}\right)$. For any $\phi^{\prime} \in V^{\prime}$, define a function $\phi$ on $D$ by $\phi(p)=\phi^{\prime}(\theta p)$. Then, this yields a linear space $V \subset C[D]$ having $\left\{D_{1}, \cdots, D_{N}\right\}$ for uniqueness domains. In particular, the sets $D_{i}$ can be selected so that their union exhausts all but a nowhere dense subset of $D$, and this can be achieved with many different choices of $V$.

This is no longer possible in general if we wish to retain the more precise inequality (1) given in Theorem 1 . Suppose that we have a space $V$ of dimension $N$, and a collection of sets $\left\{D_{i}\right\}_{1}^{N}$ such that for any $f \in V$,

$$
\|f\| \leqq B \sum_{1}^{N} \inf _{p_{i} \in D_{i}}\left|f\left(p_{i}\right)\right| .
$$

Suppose that there is a point $q$ which is a common boundary point for all the sets $D_{i}$. Then $N=1$. For, if $f \in V$ and $f(q)=0$, then by the inequality and the continuity of $f, f \equiv 0$. Suppose $\phi_{1}$ and $\phi_{2}$ are two independent functions in $V$; set $f=\phi_{2}(q) \phi_{1}-\phi_{1}(q) \phi_{2}$ and have $f(q)=0$.

UNIVERSITY OF WISCONSIN AND INSTITUTE FOR DEFENSE ANALYSES 